\title{
Propriedades da fibrina rica em plaquetas (PRF) aplicada a cirurgia oral - protocolo
}

\section{Choukroun}

\author{
Properties of platelet-rich fibrin (PRF) applied to oral surgery - Choukroun protocol \\ Propiedades de la fibrina plaquetaria (PRF) aplicada a cirugía oral - protocolo Choukroun
}

Recebido: 22/04/2021 | Revisado: 03/05/2021 | Aceito: 05/05/2021 | Publicado: 07/05/2021

\author{
Keven de Assis Fursel \\ ORCID: https://orcid.org/0000-0003-1121-7910 \\ Universidade Paulista, Brasil \\ E-mail: kevenfursel@hotmail.com \\ José Lopes de Oliveira Neto \\ ORCID: https://orcid.org/0000-0001-8181-6930 \\ Universidade Paulista, Brasil \\ E-mail: neto.lopes_@hotmail.com \\ Mateus José de Sousa \\ ORCID: https://orcid.org/0000-0003-0410-6833 \\ Universidade Paulista, Brasil \\ E-mail: mateus_jose18@hotmail.com \\ Victor Hugo Lopes de Oliveira Moreira \\ ORCID: https://orcid.org/0000-0003-4043-9344 \\ Universidade Federal de Goiás, Brasil \\ E-mail: victorhugolopes52@gmail.com \\ Rubens Jorge Silveira \\ ORCID: https://orcid.org/0000-0002-3234-2158 \\ Universidade Paulista, Brasil \\ E-mail: rubesnjs30@hotmail.com
}

\begin{abstract}
Resumo
O desenvolvimento de aditivos cirúrgicos bioativos para regular a inflamação e aumentar a velocidade do processo de cicatrização é um dos grandes desafios da pesquisa clínica. Pois em procedimentos cirúrgicos muitas vezes utilizavam técnicas que, na maioria das vezes, eram bem invasivas e causavam desconforto ao paciente. Nesse sentido, a fibrina rica em plaquetas (PRF) surge como uma alternativa natural, viável e bastante satisfatória com resultados favoráveis e apresentando baixos riscos. O PRF (Fibrina Rica em Plaquetas) foi desenvolvido por Choukroun para uso em cirurgia oral e maxilofacial, e tem diversas áreas de aplicação dentro da odontologia, podendo citar o aumento ósseo na implantodontia, levantamento de seio maxilar, transplante alveolar, cirurgia periodontal estética, dentre outros. A revisão a seguir trata-se de um estudo de revisão de literatura sobre a propriedades da fibrina rica em plaquetas (PRF) aplicada a cirurgia oral-protocolo choukroun, com base em 24 artigos selecionados de 40 artigos pesquisados enfocando na sua preparação, vantagens e desvantagens de usá-lo em aplicações clínicas, especialmente seu uso na cirurgia oral.
\end{abstract}

Palavras-chave: Plaquetas sanguíneas; Fibrina; Fibrina rica em plaquetas; Regeneração óssea; Cirurgia bucal.

\begin{abstract}
The development of bioactive surgical additives to regulate inflammation and increase the speed of the healing process is one of the great challenges of clinical research. Because in surgical procedures they often used techniques that, in most cases, were very invasive and caused discomfort to the patient. In this sense, platelet-rich fibrin (PRF) appears as a natural, viable and quite satisfactory alternative with favorable results and presenting low risks. The PRF (Fibrin Rich in Platelets) was developed by Choukroun for use in oral and maxillofacial surgery, and has several areas of application within dentistry, which can mention the bone increase in implantology, maxillary sinus lifting, alveolar transplantation, aesthetic periodontal surgery, among others. The following review is a literature review study on the properties of platelet-rich fibrin (PRF) applied to oral surgery-choukroun protocol, based on 24 selected articles from 40 researched articles focusing on its preparation, advantages and disadvantages of using it in clinical applications, especially its use in oral surgery.
\end{abstract}

Keywords: Blood platelets; Fibrin; Platelet-rich fibrin; Bone regeneration; Oral surgery.

\section{Resumen}

El desarrollo de aditivos quirúrgicos bioactivos para regular la inflamación y acelerar el proceso de curación es uno de los grandes retos de la investigación clínica. En los procedimientos quirúrgicos, a menudo utilizaban técnicas que, en la mayoría de los casos, eran muy invasivas y causaban molestias al paciente. En este sentido, la fibrina rica en 
plaquetas (PRF) aparece como una alternativa natural, viable y bastante satisfactoria con resultados favorables y de bajo riesgo. O PRF(Fibrina Rica em Plaquetas) foi desenvolvido por Choukroun para uso em cirurgia oral e maxilofacial, e tem diversas áreas de aplicação dentro da odontologia, podendo citar o aumento ósseo na implantodontia, levantamento de seio maxilar, transplante alveolar, cirurgia periodontal estética, entre otros. La siguiente revisión es un estudio de revisión de la literatura sobre las propiedades de la fibrina rica en plaquetas (PRF) aplicada al protocolo de cirugía oral-choukroun, basado en 24 artículos seleccionados de 40 artículos investigados que se centran en su preparación, ventajas y desventajas de su uso en aplicaciones clínicas, especialmente su uso en cirugía oral.

Palabras clave: Plaquetas sanguíneas; Fibrina; Fibrina rica en plaquetas; Regeneración óssea; Cirugía oral.

\section{Introdução}

Um dos principais problemas da ciência clínica é a produção de aditivos cirúrgicos bioativos que controlam a inflamação e aumentam o ritmo do processo de cicatrização. Dessa forma, a cura é um mecanismo dinâmico que envolve a coordenação de células, sinais químicos e matriz de reparação de tecidos extracelulares. O mecanismo de cura ainda está incompleto, mas as plaquetas desempenham um papel significativo na hemostasia e na cicatrização da ferida.

$\mathrm{Na}$ década de 1970, a capacidade regenerativa das plaquetas foi estabelecida quando foram detectados fatores de crescimento para uma melhora no desenvolvimento de colágeno, mitose celular, formação de vasos sanguíneos, migração de células recrutadas para o local da ferida. O uso do concentrado plaquetário para aplicações na engenharia de tecidos in vivo é um dos novos avanços na operação oral, sendo a plaqueta rica em plasma (PRP) e a plaqueta rica em fibrina (PRF). O concentrado plaquetário é constituído por fatores de crescimento que podem ser usados localmente para intensificar o processo de cicatrização de feridas como um agente cirúrgico biologicamente ativo (Kiran, Mukunda \& Tilak Raj, 2011).

Ao usar a plaqueta rica em plasma na cirurgia oral, encontrou um benefício claro, que era a capacidade de estimular o reparo e formação óssea. No entanto, o uso de trombina bovina que é utilizada no protocolo do PRP é muitas vezes perigoso, uma vez que anticorpos $\mathrm{V}$, XI, e fatores de trombina, que contribuem para a coagulopatia, podem se formar.

Em 2001, o PRF foi usado pela primeira vez por Choukroun, especificamente em cirurgia oral e maxilofacial, sendo chamado de forma moderna de plaquetas. Se consiste em uma matriz fibrina autóloga que tem muitos benefícios em comparação com o PRP, incluindo melhor armazenamento e entrega de hemoterapia química (Choukroun et al., 2006).

A revisão a seguir tem como objetivo resumir a literatura relacionada sobre a técnica de utilização da fibrina rica em plaquetas (PRF), concentrando-se em seu planejamento, vantagens e desvantagens de seu uso em aplicações clínicas, especialmente na implantodontia.

\section{Metodologia}

Para este trabalho, foi utilizado revisão bibliografica sobre propriedades da fibrina rica em plaquetas (PRF) aplicada a cirurgia oral-protocolo choukroun.

A vantagem de se realizar uma pesquisa bibliográfica está no fato de se poder abordar um fenômeno a partir de diversas perspectivas, por meio de diferentes autores, seu uso exploratório também se destaca por que a partir deste tipo de pesquisa, é possível se observar se determinado fato ou técnica possui resultados positivos de efetividade (Gil, 2002).

Deste modo, a pesquisa assume uma característica qualitativa, que conforme Gil (2002), possui um caráter descritivo dos fenômenos observados, o que deixa de lado a quantificação, com foco na análise dos conteúdos encontrados nos diversos autores pesquisados.

Foram 27 artigos selecionados de 40 artigos pesquisados com os descritores: Plaquetas sanguíneas, fibrina, fibrina rica em plaquetas, regeneração óssea, cirurgia oral. Com tudo se possibilitou a relação de palavras-chaves utilizadas na busca dos materiais necessários para o desenvolvimento da presente revisão, onde foram obtidos valiosos artigos/obras bibliográficas 
com suma importância acadêmica, vindo a buscar excelência na pesquisa, resultados importantes e relevantes para a escrita do mesmo.

\section{Resultados}

O PRF consiste em uma matriz autóloga de fibrina rica em leucócitos e plaquetas, composta por uma estrutura trimolecular, com citocinas, plaquetas, e células-tronco, que age como uma estrutura biodegradável, sendo favorável ao desenvolvimento da microvascularização orientando a migração de células epiteliais para sua superfície. Além disso, o PRF pode ser usado como um transportador para células envolvidas na regeneração de tecidos, e parece ser capaz de liberar fatores de crescimento continuamente em 1 a 4 semanas, estimulando assim o ambiente de cicatrização de feridas. Vindo a ter uma estrutura complexa de uma matriz de fibrina forte, com boas propriedades mecânicas e se remodela lentamente, semelhante a um coágulo sanguíneo (Wu et al., 2012).

Esta matriz autóloga tem mostrado grande potencial em estudos in vitro com o objetivo de aumentar a ligação celular e estimular a produção de osteoblastos.

O PRF tem propriedades imunológicas e antimicrobianas, portanto, pode levar à degranulação de leucócitos e tem certas citocinas que podem induzir angiogênese e respostas pró / anti-inflamatórias (Saluja, Dehane \& Mahindra, 2011)

A divergência entre o coágulo sanguíneo natural e o PRF, é que o último é mais uniforme e estável, sendo mais fácil de manusear e colocar em locais designados (Simonpieri et al., 2012).

Na cirurgia, pode-se utilizar o PRF como uma membrana absorvível para guiar a regeneração óssea, evitando a migração de células indesejáveis para o defeito ósseo e proporcionando um espaço que permite a imigração de células osteogênicas e angiogênicas. No entanto, uma membrana PRF normalmente tem degradabilidade rápida de uma a duas semanas, mas se as fibras forem reticuladas, pode fornecer resistência contra a degradação enzimática, assim sendo mais estável durante o tempo de cicatrização. Com o exposto acima, em pesquisa in vitro com camundongos, sugeriram compressão térmica da membrana PRF em casos com indícios de regeneração óssea guiada, pois esse procedimento é uma técnica menos citotóxica, que reduz a área superficial e a porosidade, retardando sua degradação em até 4 semanas (Kawase et al., 2015).

A concepção de "Natural Bone Regeneration" (NBR), inclui a regeneração do volume ósseo e do tecido gengival através da membrana PRF. O mesmo autor relatou resultados clínicos satisfatórios, os quais foram relacionados à remodelação de todo o osso alveolar e à recuperação do volume gengival e do osso ao redor do implante, obtendo propriedades mecânicas e estéticas suficientes (Simonpieri et al., 2012).

A membrana PRF tem apresentado resultados clínicos favoráveis no tratamento de defeitos intraósseos periodontais, protegendo feridas abertas do meio oral quando a sutura não consegue ligar as margens da mucosa e acelerando a cicatrização de tecidos duros e moles. Alguns estudos clínicos utilizaram a membrana PRF como único material de enxertia para obtenção de aumento do assoalho do seio maxilar, apresentando resultados promissores (Choukroun et al., 2006).

A membrana PRF ajuda na cicatrização de feridas, e a proteger o local da cirurgia promovendo o reparo de tecidos moles. Quando misturado com enxertos ósseos, pode atuar como um conector biológico para atrair células tronco, e promover a migração de células progenitoras ósseas para o centro do enxerto fornecendo a formação de novos vasos sanguíneos

Histologicamente e estereologicamente, o autor comparou os efeitos de cura de $\beta$-TCP e PRF, sozinhos e combinados, em defeitos ósseos padronizados em tíbias de porco (Yilmaz et al., 2014). Os resultados mostraram que quando $\beta$-TCP e PRF foram usados juntos, o osso recém-formado foi significativamente maior do que quando usado ambos separadamente. O PRF pode atuar também como um bioadesivo para manter as partículas juntas, ajudando assim a manipular o enxerto ósseo (Yilmaz et al., 2014).

Em seu estudo Tchemra e colaboradores (2021), comprovaram a efetividade da utilização do PRF como biometerial, a 
partir de um estudo de caso, no qual foi realizado um enxerto ósseo com a associação de xenógeno com PRF, em que se percebeu maior aumento do seio maxilar, ao se comparar este enxerto com o enxerto a partir do osso apenas, foi possível constatar de modo comparativo a efetividade do PRF, no caso, associado ao xenógeno.

O PRF também pode ser usado em alvéolos após extrações, como material de enxerto, atuando como um coágulo de sangue estável favorecendo a regeneração tecidual. Podendo ser aplicado para melhorar a cicatrização de feridas em pacientes imunocomprometidos e diabéticos. Além disso, como o PRF estimula a coagulação com trombospondina e o fechamento da ferida, o mesmo pode ser usado como um adjuvante em pacientes em terapia anticoagulante (Corso, Toffler, David e Ehrenfest, 2010).

Esse efeito de cicatrização, também é descrito por Kerhwald et al. (2021), que aponta melhora na regeneração de tecidos, tanto moles quanto os ósseos, o que acaba por facilitar a cicatrização, reduzindo dores e desconforto pós-operatório, em que se observou resultados favoráveis do uso do PRF na regeneração de tecidos da gengiva e ósseo.

Alguns estudos também mostram o uso do PRF em combinação com beta fosfato tricálcico, sem enxerto ósseo em procedimentos de elevação do seio nasal e lesões periodontais crônicas. O enchimento de cavidades de avulsão com PRF leva a resultados muito favoráveis quando as paredes ósseas estão intactas.

Uma combinação de PRF com substitutos ósseos e outros adjuvantes podem ser necessária em defeitos residuais onde uma ou várias paredes estão faltando ou danificadas, a fim de fornecer uma reconstrução adequada do volume ósseo. O PRF aumenta a coesão entre os materiais do enxerto, pois a fibrina atua como uma cola fisiológica entre os tecidos da ferida (Corso, Toffler, David \& Ehrenfest, 2010).

A coagulação natural do sangue leva à formação de uma matriz de fibrina que liga biologicamente o tecido ferido junto com a proliferação celular, migração celular, aposição de neomatriz e remodelamento. Portanto, a combinação de PRF com outros materiais de enxerto melhorara integração do material de enxerto, uma vez que PRF é um coágulo de sangue otimizado. Em casos de cavidades largas e lesões onde o fechamento primário é difícil, o PRF pode ser usado como uma membrana de cobertura e proteção que promove a reepitelização do local e acelera a fusão das margens da ferida (Gupta, Bains, Singh, Mathur \& Bains, 2011).

A membrana de fibrina possui elasticidade e resistência, o que facilita a sutura. Como uma membrana para regeneração óssea guiada, devido a sua arquitetura de matriz densa, o PRF cobre, protege e estabiliza o material de enxerto ósseo e o local operatório em geral (Gupta, Bains, Singh, Mathur \& Bains, 2011).

A principal vantagem do PRF é que ele tem um protocolo de preparação simples. O sangue é coletado do paciente usando um vacutainer de $10 \mathrm{ml}$ estéril (dois a 12 tubos) antes ou durante a cirurgia (Ali, Bakry \& Abd-Elhakam, 2015).

Os tubos com amostras de sangue coletadas são imediatamente (dentro de 2 minutos após a coleta da amostra de sangue) colocados na centrífuga e processados em uma única etapa de centrifugação. O sucesso clínico do protocolo de PRF depende de uma rápida coleta de sangue e sua transferência para a centrífuga, pois o sangue começará a coagular automaticamente após 1-2 minutos e dificultará a obtenção da qualidade de coágulo necessária (Ali, Bakry \& Abd-Elhakam, 2015). O não preparo rápido da PRF pode causar polimerização difusa da fibrina, o que não é ideal para a cicatrização do tecido.

Os tubos devem ser sempre balanceados opondo-se dois tubos para equilibrar as forças de centrifugação e evitar vibrações durante o processo de centrifugação (Aroca, Keglevich, Barbieri, Gera \& Etienne, 2009).

No final da centrifugação, as tampas para A-PRF ou L-PRF (não i-PRF) são removidas e os tubos colocados em um suporte de tubo estéril. A amostra de sangue com o coágulo é deixada em repouso/maturação por aproximadamente 4 a 8 minutos antes de extrair o coágulo do tubo (Aroca, Keglevich, Barbieri, Gera \& Etienne, 2009).

O processo de centrifugação ativa o processo de coagulação e separa a amostra de sangue em três camadas diferentes: 
um plasma acelular no topo do tubo; um coágulo de fibrina fortemente polimerizado é formado no meio; e as células sanguíneas (base do corpúsculo vermelho) são reunidas no fundo do tubo (Aroca, Keglevich, Barbieri, Gera \& Etienne, 2009).

Dados de pesquisa sugerem que o tipo de tubo usado (ou seja, vidro seco ou tubos de plástico revestido de vidro) e o processo de compressão do coágulo (forçado ou mole) não parecem influenciar a arquitetura deste biomaterial autólogo (Tatullo et al., 2012).

No entanto, ambos os parâmetros podem influenciar o conteúdo do fator de crescimento e as propriedades da matriz do produto (Tatullo et al., 2012). A influência desses fatores de preparação requer análises adicionais e seu efeito na eficácia clínica deve ser validado com ensaios clínicos de boa qualidade.

Cada coágulo de fibrina concentra a maioria das plaquetas (97\%) e mais da metade dos leucócitos de uma colheita de sangue de $9 \mathrm{ml}$ (Tatullo et al., 2012).

O coágulo PRF é removido do tubo com uma pinça estéril. O coágulo de fibrina é separado do fragmento de hemácias, aproximadamente $2 \mathrm{~mm}$ abaixo da linha divisória, por meio de tesoura (Tatullo et al., 2012).

A seção do coágulo de sangue anexado ao coágulo de fibrina contém as células-tronco. Os coágulos PRF são colocados na grade Box (Processo, França) ou no Kit Xpression ${ }^{\mathrm{TM}}$ (Intra-Lock, Boca Raton, Flórida) e cobertos com a tampa. As membranas PRF estão prontas para uso após 2 minutos. Ele fornece uma matriz tridimensional de plaquetas, leucócitos e fatores de crescimento (Tatullo et al., 2012).

Uma membrana PRF permanece utilizável muitas horas após a preparação, desde que a PRF seja preparada corretamente e conservada em condições fisiológicas. Além disso, o uso do PRF Box, uma ferramenta fácil de usar e barata, permite a preparação padronizada de membranas PRF homogêneas com um maior teor de fator de crescimento, evita a desidratação ou morte dos leucócitos que vivem no coágulo PRF e também previne o encolhimento da arquitetura da matriz de fibrina (Simonpieri et al., 2012).

Para a padronização de preparações de PRF como material de enxerto para regeneração de tecidos, as membranas de PRF devem ser sempre preservadas em um ambiente de soro úmido. O procedimento de compressão dos coágulos em membranas é realizado com uma pressão suave, lenta e homogênea para evitar a compressão de todo o soro contido no coágulo PRF original (Bolukbasi, Ersanli, Keklikoglu, Basegmez \& Ozdemir, 2015; Toffler, Toscano \& Holtzclaw, 2010).

As membranas PRF são fáceis de colocar sobre um local cirúrgico ou aumentado. A consistência elástica da membrana PRF também permite que o cirurgião-dentista faça um orifício na membrana para cobrir um pilar de cicatrização antes de suturar o retalho a mistura de osso autógeno ou substitutos de osso com i-PRF (PRF lquid) para uso em procedimentos de GBR transforma o osso particulado em uma consistência de gel fácil de manusear (Peck, Marnewick \& Stephen, 2011).

O PRF líquido (i-PRF) pode ser injetado acima, ou membrana PRF (A-PRF ou L-PRF) colocada acima da membrana GBR ou GTR para atuar como uma barreira de interposição para proteger e estimular o compartimento ósseo, e como uma membrana cicatrizante com o objetivo de melhorar a cicatrização e remodelação dos tecidos moles, evitando assim a deiscência dos tecidos moles (Peck, Marnewick \& Stephen, 2011).

A membrana PRF também pode ser usada como uma barreira protetora para selar e promover a cura das comunicações oroantrais após extrações (Magremanne, Baeyens, Awada \& Vervaet, 2009); para fechar um local de colheita de tecido conjuntivo palatal (Zhao, Tsaim Chang, 2011); ou como enxerto de sola em elevações do seio da face ou como curativo de vestibuloplastia (Simonpieri et al., 2012).

O PRF (membrana ou líquido) pode ser adicionado ou misturado a substitutos ósseos, como xenoenxerto ou fosfato de cálcio bifásico (BCP), para potencializar a formação de novo osso (Toeroek \& Dohan, 2010). A pesquisa sugere que a consolidação óssea é mais eficaz quando o PRF é misturado com osso autógeno ou substitutos ósseos como DBBG e BCP em procedimentos de aumento ósseo ou GBR (VijayalakshmiI, Rajmohan, Deepalakshmi \& Sivakami, 2012). 
Membranas PRF são frequentemente utilizadas para proteção da área enxertada e como reforço de cicatrização dos tecidos moles acima dos defeitos enxertados ou sítios aumentados (Inchigolo et al., 2010). A finalidade da membrana PRF não é apenas proteger o coágulo sanguíneo e / ou o material do enxerto, como no conceito de GTR, mas também promover a indução de um periósteo e gengiva fortes e espessos. Esse periósteo reforçado funciona como uma verdadeira barreira entre os tecidos moles e os compartimentos ósseos, e constitui provavelmente a melhor barreira de proteção e regeneração para os defeitos intra-ósseos (Inchigolo et al., 2010).

A preparação do PRF é fácil, rápida e fácil de usar na rotina clínica diária. O PRF é preparado no local (no ambiente clínico) simplesmente retirando sangue do paciente para uso imediato, reduzindo assim o tempo de espera do paciente. Os protocolos usados atualmente para a preparação de PRF autóloga são padronizados e fáceis de usar pelos médicos e assistentes clínicos. Além disso, não há limitação da quantidade de membranas PRF necessárias (Inchigolo et al., 2010).

Em comparação com um coágulo de sangue natural, o PRF é um material sólido, tem uma arquitetura de fibrina forte e mais fácil de manusear e posicionar. A arquitetura tridimensional da fibrina fornece à membrana PRF grande densidade, elasticidade, flexibilidade e força que é mais adequada para manipulação e sutura (Hauser et al, 2013). A consistência elástica da membrana PRF permite que o clínico a perfure para permitir sua colocação ao redor de uma cicatrização ou pilar protético ou mesmo sutura.

O sangue é coletado do paciente e, portanto, a morbidade do local doador é reduzida. PRF raramente causa complicações como exposição da membrana; um resultado indesejado que foi observado em casos que usam membranas de barreira biodegradáveis. Outra vantagem da PRF é o risco extremamente baixo de infecção. Além disso, nenhum efeito de citotoxicidade in vitro foi detectado, independentemente da quantidade de PRF utilizada.

Tem uma excelente relação custo-benefício. Uma membrana de fibrina rica em plaquetas (PRF) é um biomaterial prontamente disponível e barato que é benéfico em implantodontia, cirurgia oral e procedimentos periodontais (Bajaj et al., 2013).

A facilidade de preparação e o custo-benefício da membrana PRF oferece uma grande vantagem sobre outras membranas disponíveis comercialmente. É amplamente aplicável na odontologia, sendo financeiramente realista para o paciente e o sistema de saúde. PRF é atualmente a escolha mais segura e econômica para pacientes e médicos para melhorar os resultados de cicatrização e regeneração de tecidos (tecidos moles e ossos) (Bajaj et al., 2013).

Pode poupar o paciente de campo operatório adicional (morbidade na segunda cirurgia) e também economizar custos ao evitar o uso de membranas aloplásticas ou xenogênicas e reduzir a quantidade de materiais de enxerto sintéticos (Thorat, Pradeep \& Pallavi, 2011). É também uma alternativa econômica aos dispendiosos fatores de crescimento recombinantes quando usados em conjunto com enxertos ósseos.

As membranas PRF não têm contraindicações: podem ser usadas em todos os tipos de pacientes, especialmente em pacientes com condições sistêmicas onde a cicatrização está comprometida (ou seja, diabéticos e fumantes), ou em situações comprometidas cirurgicamente (retalho danificado). Nessas situações, a PRF irá promover a cicatrização de partes moles e estimular a cicatrização de um retalho danificado e reduzir os riscos de necrose do retalho após uma cirurgia. É um ponto comum que todos os produtos à base de fibrina (concentrados de plaquetas) são frequentemente usados para a estimulação da angiogênese e para reduzir o risco de necrose do retalho em muitas aplicações de cirurgia (Desarda, Gurav, Gaikwad \& Inamdar, 2013).

A técnica é de acesso aberto e, portanto, pode ser amplamente desenvolvida e usada na prática privada sem considerações comerciais (Toffler, Toscano \& Holtzclaw, 2010). As aplicações clínicas do PRF em outras áreas da odontologia, como a endodontia, também estão aumentando exponencialmente. A diversidade do PRF se faz tão diversa, e segura, que Lins, Brandão e Rocha apontam o seu uso extrapola o uso clínico odontológico, como por exemplo, pode-se citar 
seu uso no campo da estética, no rejuvenescimento da pele, e na melhoria da aparência de cicatrizes.

\section{Discussão}

A partir desta revisão de literatura pode-se notar, que o PRF tem sido cada vez mais estudado e utilizado por médicos e cirurgiões-dentistas em todo o mundo, como um biomaterial autólogo adjuvante que promove a cicatrização e regeneração óssea e de tecidos moles.

Segundo Choukroun et al. (2006), a aplicação de fatores biológicos em reconstruções ósseas vem sendo muito explorada, destacando os fatores de crescimento derivados de plaquetas, que tem o objetivo de otimizar os princípios biológicos obtendo melhor previsibilidade da resposta tecidual.

De acordo com Dohan et al. (2006), os concentrados de plaquetas usados tradicionalmente tinham objetivo de prevenir e tratar hemorragias, por isso eram originalmente utilizados na medicina, principalmente em hematologia.

Choukroun et al. (2006) e Dohan et al. (2006) constataram que muitos artigos científicos começaram a alegar que o derivado de plaquetas poderia ser eficaz não apenas para hemostasia, mas também na melhoria da resposta dos tecidos a enxertos.

Portanto, essa tendência de aditivos cirúrgicos deu início com a cola de fibrina, que depois evoluiu para o concentrado de plaquetas (PRP). No entanto, o uso de trombina bovina no tratamento do plasma risco em plaquetas foi considerado arriscado, levando em consideração que o fator $\mathrm{V}$ da coagulação poderia ter uma reação com o fator $\mathrm{V}$ da coagulação humana, podendo causar complicações.

Além disso, o uso do PRP na prática clínica diária se tornou complicado, devido a dificuldade de manipulação sanguínea e nos seus protocolos de preparação. Então, em 2001 Choukroun et al. (2006) descreveu a tecnologia da plaqueta rica de fibrina (PRF).

Segundo Choukroun et al. (2006) e Dohan et al. (2006), a fibrina rica em plaquetas é a segunda geração de concentrado de plaquetas, porque o sangue é coletado e imediatamente levado a centrífuga para formar o coágulo, sem anticoagulantes, trombina bovina ou outros agentes, diferente do PRP.

Em comparação com a antiga geração, o PRF exibe uma maior expressão e concentração de fatores de crescimento e proteínas da matriz, que são liberados mais lentamente devido à arquitetura tridimensional das glicoproteínas adesivas na fibrina, o que resulta em melhor desempenho.

Choukroun et al. (2006) descreve o PRF como uma matriz que contém todos os elementos moleculares e celulares para induzir a cura, sendo um biomaterial terapêutico com alto potencial de regeneração óssea e de partes moles, sem reações inflamatórias, podendo ser utilizado isoladamente ou em combinação com enxertos ósseos, promovendo hemostasia, crescimento e maturação óssea.

Portanto, baseado nesses estudos, buscas e pesquisas apresentadas e descritas neste presente trabalho, concluímos que a fibrina rica em plaquetas tem chamado cada vez mais a atenção porque é derivada do próprio sangue dos pacientes; está prontamente disponível, é simples de preparar; pode ser produzido imediatamente na cadeira; fácil de usar, praticamente sem risco de reação de rejeição resposta de corpo estranho; e amplamente aplicável em odontologia, sendo muito eficaz em cirurgia oral, e nas reconstruções para implantodontia.

\section{Conclusão}

Estudos têm demonstrado resultados seguros e promissores, sem achados contraditórios, relacionados ao uso de PRF isoladamente ou em combinação com outros biomateriais. Apresenta diversas vantagens e possíveis indicações para uso tanto 
na medicina quanto na odontologia.

Atualmente, a fibrina rica em plaquetas parece ser uma técnica minimamente invasiva aceita, com baixo risco e resultados clínicos satisfatórios.

O PRF pode ser usado como uma membrana (A-PRF ou L-PRF), gel (i-PRF), tampão ou a membrana pode ser cortada em fragmentos e aplicada em terapias autônomas; terapias aditivas; ou usado em terapias de combinação com outros biomateriais.

Mais importante, o uso de PRF permite a entrega local de uma matriz de fibrina, células, fatores de crescimento e proteínas que fornecem propriedades biológicas exclusivas e pistas para promover a formação de novos vasos sanguíneos e potencialmente acelerar a cicatrização de feridas e regeneração de tecidos, ao mesmo tempo reduzindo eventos adversos.

Consequentemente, os benefícios da PRF na cicatrização de feridas e ossos, seus efeitos antibacterianos e antihemorrágicos, os baixos riscos de seu uso e a disponibilidade de métodos de preparação fáceis e de baixo custo devem encorajar mais cirurgiões-dentistas a adotar esta tecnologia em suas práticas para o benefício de seus pacientes.

\section{Referências}

Ali, S., Bakry, S. A., \& Abd-Elhakam, H. (2015). Platelet-rich fibrin in maxillary sinus augmentation: a systematic review. J Oral Implantol, 41 (6): 746-753.

Aroca, S., Keglevich, T., Barbieri, B., Gera, I., \& Etienne, D. (2009) Clinical evaluation of a modified coronally advanced flap alone or in combination with a platelet-rich fibrin membrane for the treatment of adjacent multiple gingival recessions: a 6-month study. J Periodontol, 80 (2): $244-252$.

Bajaj, P., Pradeep, A. R., Agarwal, E., et al. (2013). Comparative evaluation of autologous platelet-rich fibrin and platelet-rich plasma in the treatment of mandibular degree II furcation defects: a randomized controlled clinical trial. J Periodont Res, 48 (5): 573-581.

Bolukbasi, N., Ersanli, S., Keklikoglu, N., Basegmez, C., \& Ozdemir, T. (2015) Sinus Augmentation With Platelet-Rich Fibrin in Combination With Bovine Bone Graft Versus Bovine Bone Graft in Combination With Collagen Membrane. J Oral Implantol, 41 (5): $586-595$.

Choukroun, J., Diss, A., Simonpieri, A., Girard, M. O., Schoeffler, C., Dohan, S. L., Dohan, A. J., Mouhyi, J., \& Dohan, D. M. (2006). Platelet-rich fibrin (PRF): a second-generation platelet concentrate. Part IV: clinical effects on tissue healing. Oral Surg Oral Med Oral Pathol Oral Radiol Endod, 101: e56-60.

Corso M. D., Toffler M., David M., \& Ehrenfest D. (2010). Use of an autologous leukocyte and platelet-rich fibrin (L-PRF) membrane in post-avulsion sites: An overview of Choukroun's PRF. O jornal de implantes e odontologia clínica avançada, 1 (9): 27-35.

Desarda, H. M., Gurav, A. N., Gaikwad, S. P., \& Inamdar, S. P. (2013). Platelet rich fibrin: a new hope for regeneration in aggressive periodontitis patients: report of two cases. Indian J Dent Res., 24: 627-630.

Gil, A. T. C. (2002). Como elaborar projetos de pesquisa. Atlas.

Gupta, V., Bains, B. K., Singh, G. P., Mathur, A., \& Bains, R. (2011). Regenerative Potential of Platelet Rich Fibrin In Dentistry: Literature Review. Asian J Oral Health Allied Sci, 1: 22-28.

Hauser, F., Gaydarov, N., Badoud, I., et al. (2013). Clinical and histological evaluation of postextraction platelet-rich fibrin socket filling: a prospective randomized controlled study. Implant Dent., 22 (3): 295-303.

Inchigolo, F., Tatullo, M., Marrelli, M., et al. (2010). Trial with Platelet-Rich Fibrin and Bio-Oss used as grafting materials in the treatment of the severe maxillar bone atrophy: clinical and radiological evaluations. Eur Rev Med Pharmacol Sei., 14 (12): 1075-1084.

Kawase, T., Kamiya, M., Kobayashi, M., Tanaka, T., Okuda, K., Wolff, L. F., \& Yoshie, H. (2015). The heat-compression technique for the conversion of platelet-rich fibrin preparation to a barrier membrane with a reduced rate of biodegradation. J Biomed Mater Res B Appl Biomater, 103: 825-31.

Kerhwald, R., et al. (2021). Uso de fibrina rica em plaqueta em enxerto ósseo e implantes dentários, Research, Society and Development, 10 (1): e56510112210. Doi: http://dx.doi.org/10.33448/rsd-v10i1.12210

Kiran, N. K., Mukunda, K. S., \& Tilak Raj, T. N. (2011). Platelet concentrates: A promising innovation in dentistry. J Dent Sci Res, 2 : 50-61.

Lins, V. F., Brandão, D. G., \& Rocha, S. M. W. (2021). A utilização da fibrina rica em plaquetas em procedimentos estéticos orofaciais: uma revisão integrativa. Research, Society and Development, 10 (3): e27910313477. Doi: http://dx.doi.org/10.33448/rsd-v10i3.13477

Magremanne, M., Baeyens, W., Awada, S., \& Vervaet, C. (2009). Solitary bone cyst of the mandible and platelet rich fibrin (PRF). Rev Stomatol Chir Maxillofac., 110 (2): 105-108.

Peck, M. T., Marnewick, J., \& Stephen, L. (2011) Alveolar Ridge Preservation Using Leukocyte and Platelet-Rich Fibrin: A Report of a Case. Case Rep Dent., 345048 .

Saluja, H., Dehane, V. \& Mahindra, U. (2011). PlateletRich fibrin: Platelet-Rich fibrin: A second generation platelet concentrate and a new friend of oral and maxillofacial surgeons. Ann Maxillofac Surg., 1: 53-57. 
Research, Society and Development, v. 10, n. 5, e59510515338, 2021

(CC BY 4.0) | ISSN 2525-3409 | DOI: http://dx.doi.org/10.33448/rsd-v10i5.15338

Simonpieri, A, Del Corso M, Vervelle A, Jimbo R, Inchingolo F, Sammartino G., \& Dohan Ehrenfest D. M. (2012). Current knowledge and perspectives for the use of platelet-rich plasma (PRP) and platelet-rich fibrin (PRF) in oral and maxillofacial surgery part 2: Bone graft, implant and reconstructive surgery. Curr Pharm Biotechnol, 13: 1231-1256.

Tatullo M, Marrelli M, Cassetta M, Pacifici A, Stefanelli LV, Scacco S, Dipalma G, Pacifici L \& Inchingolo F. (2012). Platelet Rich Fibrin (P.R.F.) in Reconstructive Surgery of Atrophied Maxillary Bones: Clinical and Histological Evaluations. Int J Med Sci., 9: 872-880.

Tchemra, F. G. C., et al. (2021). Efetividade do uso da Fibrina Rica em Plaquetas (PRF) no levantamento de seio maxilar: relato de caso. Research, Society and Development, 10 (1): e29210111492. Doi: http://dx.doi.org/10.33448/rsd-v10i1.11492

Thorat, M. K., Pradeep, A. R., \& Pallavi, B. (2011). Clinical effect of autologous platelet-rich fibrin in the treatment of intra-bony defects: a controlled clinical trial. J Clin Periodontol., 38: 925-932.

Toeroek, R., \& Dohan, E. D. M. (2013). The concept of Screw-Guided Bone Regeneration (S-GBR). Part 3: fast screw-guided bone regeneration (FS-GBR) in the severely resorbed preimplant posterior mandible using allograft and leukocyte- and platelet-rich fibrin (L-PRF): a 4-year follow-up. POSEIDO, 1 (2): 93100 .

Toffler M, Toscano N \& Holtzclaw D. (2010). Osteotome-Mediated Sinus Floor Elevation Using Only Platelet-Rich Fibrin: An Early Report on 110 Patients. Implant Dent., 19: 447-456.

VijayalakshmiI, R, Rajmohan, C. S., Deepalakshmi, D., \& Sivakami, G. (2012). Use of platelet rich fibrin in a fenestration defect around an implant. J Indian Soc Periodontol., 16 (1): 108-112.

Wu, C. L., Lee, S. S., Tsai, C. H., Lu, K. H., Zhao, J. H., \& Chang, Y. C. (2012). Platelet-rich fibrin increases cell attachment, proliferation and collagenrelated protein expression of human osteoblasts. Aust Dent J., 57: 207-212.

Yilmaz, D., Dogan, N., Ozkan, A., Sencimen, M., Ora, B. E., \& Mutlu, I. (2014). Effect of platelet rich fibrin and beta tricalcium phosphate on bone healing. A histological study in pigs. Acta Cir Bras, 29: 59-65.

Zhao, J. H., Tsai, C. H., \& Chang, Y. C. (2011). Clinical and histologic evaluations of healing in an extraction socket filled with platelet-rich fibrin. J Dent Sci. 2011, 6 (2): 116-122. 